\title{
Developmental Profile of Annexin IX and its Possible Role in Programmed Cell Death of the Bombyx mori Anterior Silk Gland
}

\author{
Yu Kaneko, Keiko Takaki, Masafumi Iwami and Sho Sakurai* \\ Division of Life Sciences, Graduate School of Natural Science and Technology, \\ Kanazawa University, Kakumamachi, Kanazawa 920-1192, Japan
}

\begin{abstract}
During pupal metamorphosis, the anterior silk gland (ASG) of the silkworm, Bombyx mori, undergoes programmed cell death (PCD), which is triggered by 20-hydroxyecdysone (20E). Annexin IX $(A N X I X)$ has been identified as a 20E-inducible gene in dying ASGs, and we show here that its expression is down-regulated in tissues destined to die but not in tissues that survive pupal metamorphosis. ANX IX expression was high in the ASGs during the feeding period, when the ecdysteroid titer was low, and decreased in response to the rising ecdysteroid titer that triggered pupal metamorphosis. Before gut purge, in vitro exposure of the ASGs to 20E levels corresponding to the ecdysteroid concentration present at the time of gut purge caused a decrease in ANX IX messenger RNA levels. Expression profiles of EcR and USP, and the 20E concentration-responses of these genes, indicate the importance of the relative abundance of $E c R-A$ and $E c R-B 1$ isoforms in $A N X$ IX regulation. These results suggest an involvement of ANX IX in the determination of PCD timing by delaying or suppressing the response to the increase in hemolymph ecdysteroid concentration during the prepupal period.
\end{abstract}

Key words: annexin, Bombyx, 20-hydroxyecdysone, $E c R$, programmed cell death

\section{INTRODUCTION}

Programmed cell death (PCD), an evolutionarily conserved process of cell suicide, is used to selectively remove unwanted cells in multicellular animals and plays an important role in development (Jacobson et al., 1997). For example, during insect larval-pupal metamorphosis, the insect steroid hormone 20-hydroxyecdysone (20E) triggers PCD to remove larval-specific tissues. The molecular mechanisms of PCD have been extensively studied in the fruit fly Drosophila melanogaster (Abrams, 1999). In Drosophila salivary glands, 20E has been shown to induce the hierarchical expression of the ecdysone-regulated genes $E c R$, ultraspiracle (USP), E74, broad-complex (BR-C), $\beta F T Z-F 1$, and E93, followed by activation of caspases (Jiang et al., 2000; Lee and Baehrecke, 2001; Yin and Thummel, 2005).

The silk gland of the silkworm Bombyx mori is a larvalspecific tissue that undergoes PCD at larval-pupal metamorphosis. It is divided into three parts: the anterior silk gland (ASG), middle silk gland (MSG), and posterior silk gland (PSG). The MSG and PSG produce large amounts of the silk proteins sericin and fibroin, respectively, while the ASG is a duct composed of a cell monolayer from which silk filaments are formed out of the liquid silk proteins.

Bombyx mori fifth instar ASGs obtained on the day of

\footnotetext{
* Corresponding author. Phone: +81-76-264-6250; Fax : +81-76-264-6255;

E-mail : ssakurai@kenroku.kanazawa-u.ac.jp
}

doi:10.2108/zsj.23.533 gut purge (day $7, \mathrm{~V} 7$ ) have previously been shown to respond to 20E while undergoing PCD (Chinzei, 1975; Terashima et al., 2000), while annexin IX (ANXIX) has been identified as a 20E-inducible gene that is up-regulated on V7 in the ASGs (Tsuzuki et al., 2001). The annexins (ANXs) are a family of structurally related calcium-dependent phospholipid-binding proteins. They contain a highly divergent amino-terminal domain that might confer unique functions to individual family members. In cultured mammalian cells, ANXs function in a wide range of physiological processes (Rescher and Gerke, 2004), such as promoting the aggregation of phospholipid membranes (Emans et al., 1993; König et al., 1998), inhibiting phospholipases (Davidson et al., 1987; Mira et al., 1997), and suppressing PCD via regulation of the $\mathrm{Ca}^{2+}$ influx (Gidon-Jeangirad et al., 1999a). Two ANX proteins have been isolated in $B$. mori: ANX b13 (Matsunaga and Fujiwara, 2002) and ANX IX (Xia et al., 2001). The three isoforms of ANX IX (ANX IX-A, ANX IX-B, and $A N X$ IX-C) contain putative $\mathrm{Ca}^{2+}$ binding sites and are generated by alternative splicing of the carboxyl-terminal exon (Xia et al., 2001).

In the ASGs of gut-purged B. mori larvae, 20E triggers PCD and up-regulates $A N X I X$ (Tsuzuki et al., 2001), suggesting that ANX IX might play a role in PCD. To address this issue, we examined the developmental profiles of $A N X$ $I X$ expression and its response to $20 \mathrm{E}$ in various tissues, in conjunction with the expression of the heterodimeric nuclear receptor genes EcR and USP. We show that ANX IX persists at high levels in tissues that survive metamorphosis, but is decreased in tissues that undergo PCD. ANX IX is 
down-regulated in ASGs by low concentrations of $20 \mathrm{E}$ corresponding to the ecdysteroid titer around the time of pupal metamorphosis. Before gut purge, ASG $A N X I X$ is incapable of responding to $20 \mathrm{E}$, but gains this ability on day 6 of photophase (V6). We show that V6 is also a critical time point for the change in isoform abundance of the heterodimeric nuclear-response-complex proteins EcR and USP, which mediate the $20 \mathrm{E}$ response.

\section{MATERIALS AND METHODS}

\section{Animals}

B. mori larvae (Kinshu $\times$ Shouwa, F1 hybrid) were reared on an artificial diet (Silkmate 2M, Nihon Nosan Kogyo, Yokohama, Japan) at $25^{\circ} \mathrm{C}$ under a $12 \mathrm{~h}$ light:12 h dark cycle (Sakurai, 1983, 1984). Under these conditions, fourth instar larvae molted during scotophase, and newly molted fifth (last) instar larvae were segregated at the start of the following photophase. The beginning of the first photophase was designated day 0 (V0). Fifth instar larvae were staged twice according to the occurrence of spinneret pigmentation at the beginning of $\mathrm{V} 6$ and the gut purge that occurred during the scotophase of V6 (gate I larvae; Sakurai et al., 1998). Gate I larvae, which pupated on V10, were used in the present study, and ASGs were dissected at around $0600 \mathrm{~h}$ on a given day.

\section{Hormones, chemicals and tissue culture}

Cycloheximide (CHX; Sigma, St Louis, MO) and 20E (Sigma) were dissolved in distilled water and stored at $-20^{\circ} \mathrm{C}$. ASGs were cultured individually in $300 \mu \mathrm{l}$ Grace's insect medium (Gibco, Grand Island, NY) at $25^{\circ} \mathrm{C}$.

\section{Determination of hemolymph ecdysteroid concentration}

Hemolymph was collected from ice-chilled female animals, and 2 or 4 volumes of methanol were added to $200-\mu$ l samples. After centrifugation at $10,000 \times g$ for $10 \mathrm{~min}$, an aliquot of supernatant was added with cyclohexane, vortexed, and centrifuged at 10,000 $\times g$ for $10 \mathrm{~min}$. An aliquot of the resulting lower phase was evaporated, dissolved in $10 \mu \mathrm{l}$ water, and subjected to an ecdysone radioimmunoassay (RIA) using O-6 anti-ecdysone antiserum (Sakurai et al., 1998). The biologically active ecdysteroid 20E (Gilbert et al., 1996) was used as a standard for RIA.

\section{DAPI staining and DNA laddering}

ASGs were fixed with $4 \%$ formaldehyde for $15 \mathrm{~min}$ at room temperature and washed three times with phosphate-buffered saline (PBS) comprising $137 \mathrm{nM} \mathrm{NaCl}, 2.7 \mathrm{mM} \mathrm{KCl}, 8.1 \mathrm{mM}$ $\mathrm{Na}_{2} \mathrm{HPO}_{4}$, and $1.47 \mathrm{mM} \mathrm{KH} \mathrm{PO}_{4}(\mathrm{pH} 7.4)$. They were then incubated with $0.1 \mu \mathrm{g} / \mathrm{ml}$ 4',6-diamidino-2-phenylindole dihydrochloride (DAPI; Sigma) in PBS in darkness for $15 \mathrm{~min}$, and washed three times with PBS. DAPI signals were detected using a fluorescence microscope with an ultraviolet excitation filter (BX50, Olympus, Tokyo, Japan). DNA was extracted from silk glands using a modified genomic DNA-extraction method (Sambrook and Russell, 2001) and electrophoresed on $2.0 \%$ agarose gels, followed by staining with ethidium bromide.

\section{Reverse transcription-polymerase chain reaction (RT-PCR) and real-time quantitative PCR (real-time Q-PCR)}

Total RNA was extracted from tissues by the acid guanidinium thiocyanate-phenol-chloroform method (Chomczynski and Sacchi, 1987) and then treated with RNase-free DNase I (Promega, Madison, WI). Complementary DNA (cDNA) was prepared from 0.75 or $1 \mu \mathrm{g}$ total RNA using anchored oligo-dT [5'-(T)12(A/C/G)(A/C/G/T)3'] and the reverse transcriptase ReveTra Ace (Toyobo, Osaka, Japan). For RT-PCR, ANX IX cDNA was amplified for 20 to 26 cycles with primers that were common to $A N X I X-A, A N X I X-B$, and $A N X$ IX-C (Table 1). Ribosomal protein $L 3(R p L 3)$ was used as an internal standard and amplified for 20 to 23 cycles. PCR products were separated by agarose gel electrophoresis, and individual gene transcripts were quantified on a real-time thermal cycler (Model 7700; Applied Biosystems, Foster City, CA). Serial dilutions of ANX $I X$ cDNA were used as standards, or serial dilutions of the pGEM$7 \mathrm{zf}(+)$ plasmids containing individual cDNAs of $E c R-A, E c R-B 1$, USP-1, USP-2, and RpL3. The SYBR Green PCR Core Reagents Kit (Applied Biosystems) was used for real-time Q-PCR according to the manufacturer's instructions. The molar amounts of $E c R-A$, $E C R-B 1, U S P-1, U S P-2$, and $R p L 3$ transcripts were calculated based on the crossing-point analysis, with standard curves generated from the plasmid containing individual cDNAs. Transcript levels of individual genes in a cDNA sample were normalized to the $R p L 3$ transcript level in the same sample. Table 1 summarizes primer sequences and annealing temperatures for individual genes.

\section{RESULTS}

\section{In vivo progression of $\mathrm{PCD}$}

The sequence of PCD in ASGs begins on V9, 2 days after gut purge (Terashima et al., 2000), but such timing remained to be seen in the MSGs and PSGs. We performed daily examinations of nuclear condensation, nuclear fragmentation, and DNA fragmentation to determine the day on which PCD began in MSGs and PSGs (Fig. 1). The nuclei of ASGs were highly branched throughout the cells, and their filamentous morphology did not change until V9. On V10, the nuclei appeared to be condensed, and the condensation progressed gradually until PO (Fig. 1A). In MSGs and PSGs, the nuclear morphology mirrored that of the ASGs,

Table 1. Oligonucleotide primers and annealing temperatures used for RT-PCR and real-time Q-PCR

\begin{tabular}{|c|c|c|c|c|}
\hline \multirow[t]{2}{*}{ Gene } & \multicolumn{2}{|c|}{ Primer sequences } & \multirow{2}{*}{$\begin{array}{c}\text { Annealing } \\
\text { temperature }\left({ }^{\circ} \mathrm{C}\right)\end{array}$} & \multirow{2}{*}{$\begin{array}{l}\text { References and } \\
\text { accession nos. }\end{array}$} \\
\hline & 5' primers & 3' primers & & \\
\hline ANX IX (RT-PCR) & GAATCTCAACATGAGCGGACA & CTTAGCGTGACCAACACATGC & 60 & \\
\hline $\begin{array}{l}\text { ANX IX } \\
\text { (Real-time Q-PCR) }\end{array}$ & GAATCTCAACATGAGCGGACA & TCCAATTCCTGAGACAGCATC & 63 & $\begin{array}{l}\text { Xia et al. (2001) } \\
\text { AB031208 }\end{array}$ \\
\hline EcR-A & TGGAGCTGAAACACGAGGTGGC & TCCCATTAGGGCTGTACGGACC & 60 & $\begin{array}{l}\text { Kamimura et al. (1997) } \\
\text { D87118 }\end{array}$ \\
\hline EcR-B1 & ATAACGGTGGCTTCCCGCTGCG & CGGTGTTGTGGGAGGCATTGGTA & 60 & $\begin{array}{l}\text { Kamimura et al. (1996) } \\
\text { D43943 }\end{array}$ \\
\hline USP-1 & GTCGAGCGTGGCGAAGAAA & CAGCCATTGTATATCGAGTTCAA & 64 & $\begin{array}{l}\text { Tzertzinis et al. (1994) } \\
\text { U06073 }\end{array}$ \\
\hline USP-2 & GATATCGTGATAATAAACCTAAGTA & GCAACAAGGTCGTTGAACTAA & 56 & AB182582 \\
\hline RpL3 & AGCACCCCGTCATGGGTCTA & TGCGTCCAAGCTCATCCTGC & 60 & $\begin{array}{l}\text { Matsuoka and Fujiwara } \\
(2000) \text { AB024901 }\end{array}$ \\
\hline
\end{tabular}




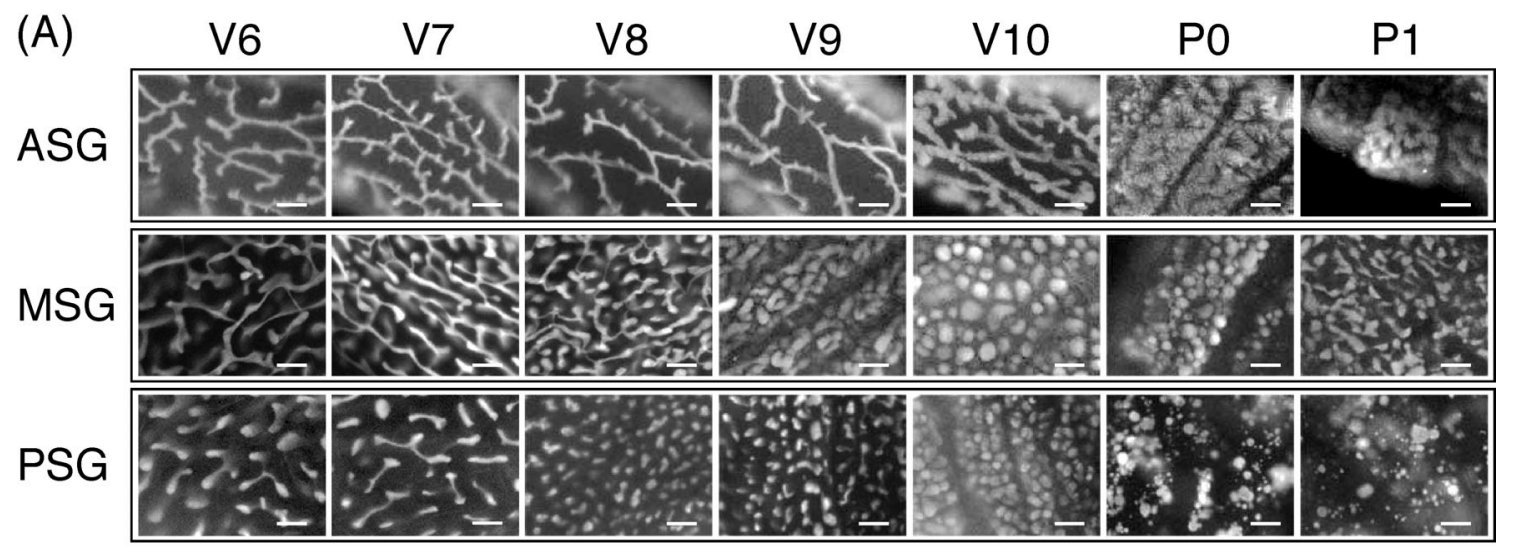

(B)

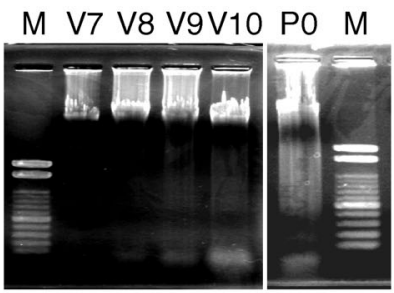

(C)

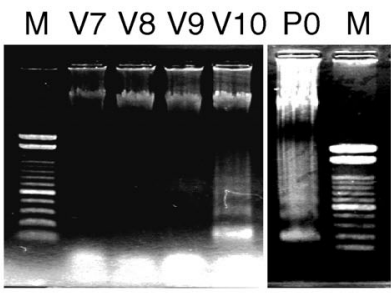

(D)

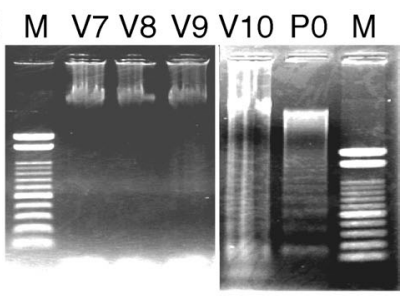

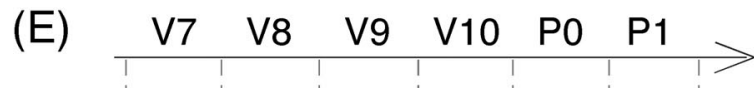

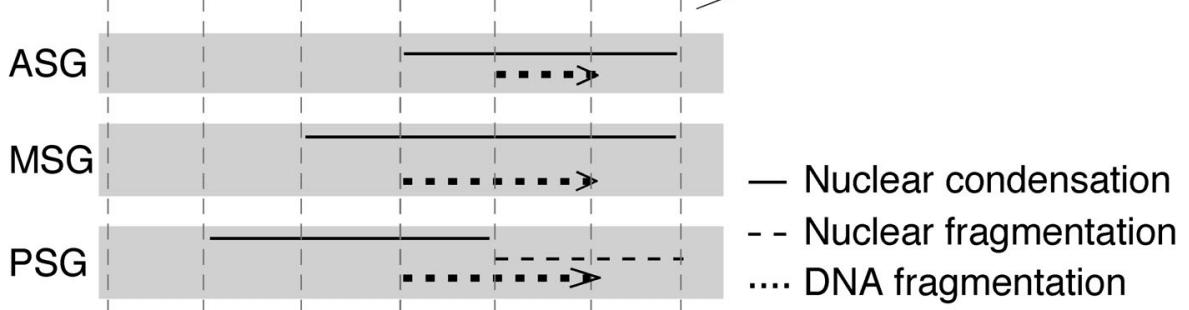

Fig. 1. In vivo progression of PCD in B. mori silk glands. Tissues were dissected out between day 6 of the fifth instar stage (V6) and 1 day after pupation (P1). (A) Changes in nuclear morphology in ASGs, MSGs, and PSGs. Scale bar, $10 \mu \mathrm{m}$. (B-D) DNA fragmentation. Genomic DNA was extracted from tissues on the indicated days and electrophoresed on agarose gels. $M=100$ base-pair ladder. (E) Schematic representation of PCD progression in ASGs, MSGs, and PSGs. Solid lines, nuclear condensation; broken lines, nuclear fragmentation; dotted lines, DNA fragmentation.

and the filamentous shape was maintained until V7. Nuclear condensation in the MSGs and PSGs occurred on V9 and V8, respectively, and nuclear fragmentation began on $P 0$ in the PSGs. Nuclear fragmentation in the ASGs and MSGs was not observed until at least $P 1$. DNA fragmentation in the ASGs commenced on P0, and was observed from V10 in the MSGs and PSGs, shortly before pupation (Fig. 1 B-D). Thus, PCD progressed from the posterior to the anterior part of the silk glands (Fig. 1E).

\section{Tissue-specific and stage-specific expression of $A N X I X$}

Silkworm ANX IX exists as three isoforms generated by alternative deletion of one or both of the second and third exons from the 3' end (Xia et al., 2001). The most 3'-end exon is found in all three isoforms, making the quantification of individual transcripts by real-time Q-PCR impossible. Accordingly, we designed primers that were common to all isoforms, and separated the PCR products by gel electrophoresis (Fig. 2A). The three isoforms were found in all tissues examined, and the expression profiles of individual isoforms within a tissue were similar to each other. The profiles were, however, tissue specific. In the ASGs, expression levels of the three isoforms were high during the feeding period, decreased on V7, and remained low until PO. The expression levels in the MSGs and PSGs decreased on V4 and V2, respectively. In both glands, levels increased transiently on V7. In Malpighian tubules and testes that survived pupal metamorphosis, high expression levels were maintained throughout the prepupal period (V7 to V10), but decreased during this time in the ASGs, MSGs, and PSGs. $A N X I X$ is thus down-regulated during the prepupal period in tissues that are destined to die, but not in those that survive pupal metamorphosis. These expression profiles suggest the possible involvement of $A N X I X$ in PCD.

Isoform abundance varied between tissues, with ANX IX-B and ANX IX-C being predominantly expressed in the ASGs. By contrast, the $C$ isoform was predominant in the MSGs and PSGs, and the $B$ isoform was predominant in the testes. This suggests that the mechanism of gene control in the ASGs might differ from that in the MSGs and PSGs, although all three parts of the silk gland are equally committed to cell death. 


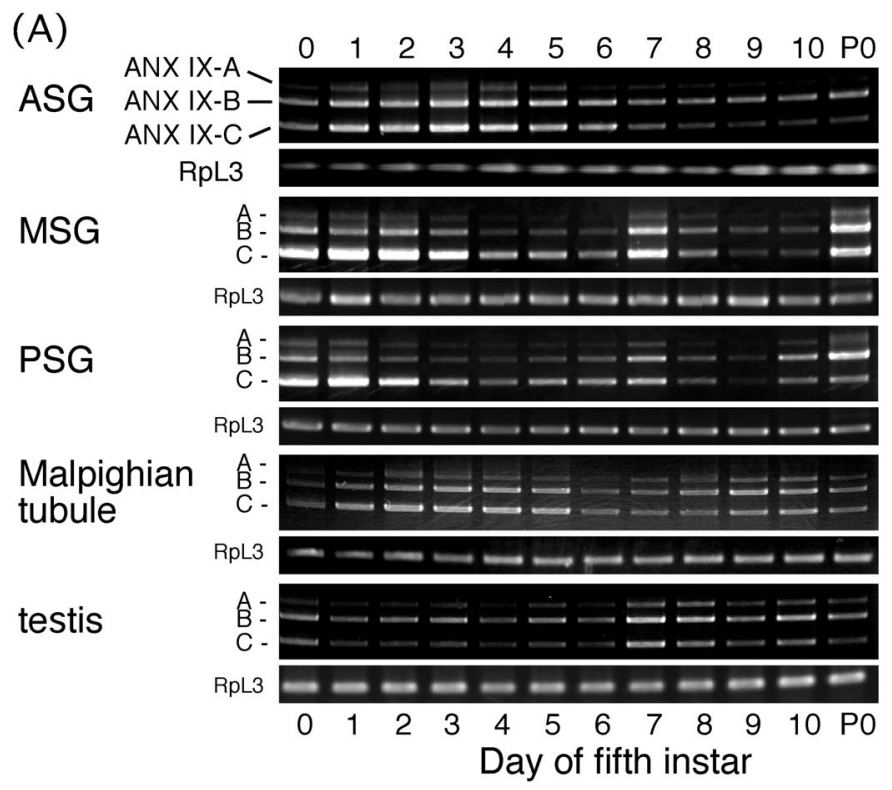

(B)
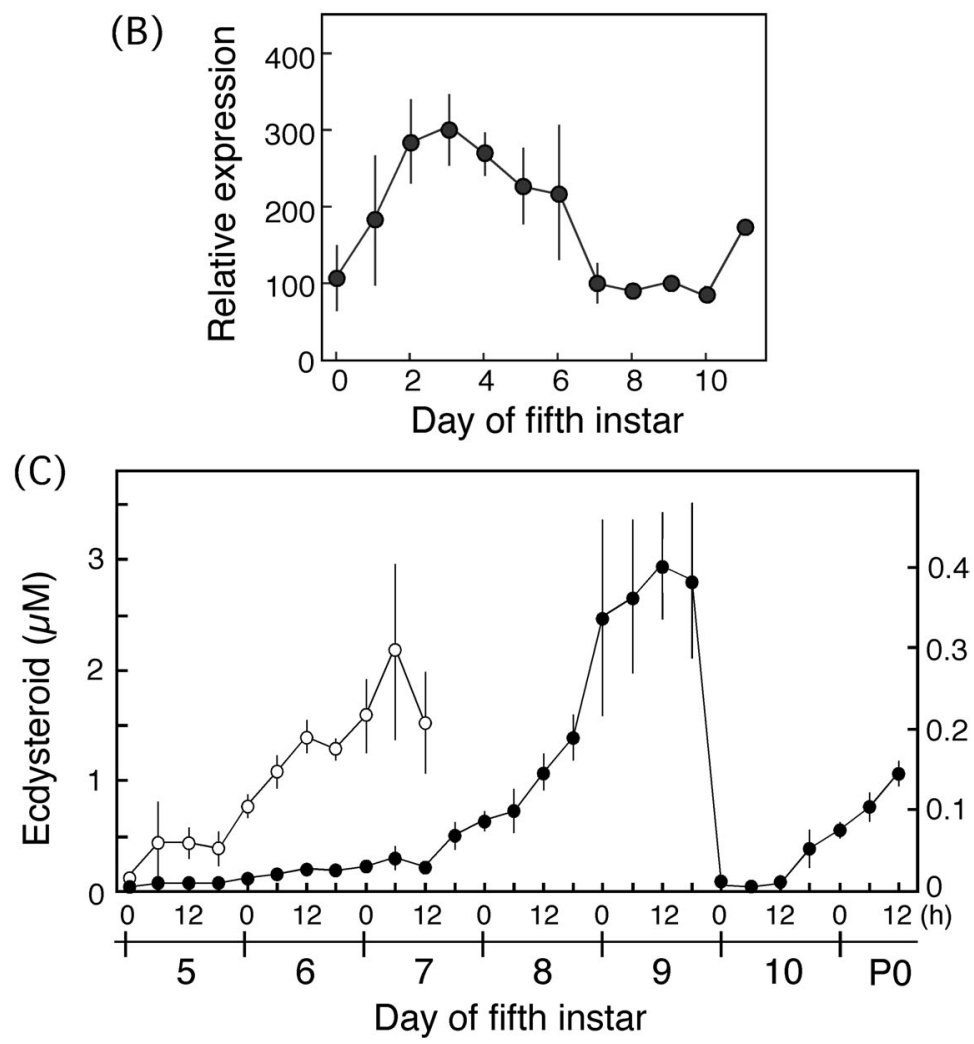

Fig. 2. Temporal expression profile of $A N X I X$ and changes in ecdysteroid titer. (A) Expression of the three ANX IX isoforms in ASGs, MSGs, PSGs, Malpighian tubules, and testis, as determined by RT-PCR. (B) Temporal expression profiles of $A N X I X$ in ASGs, as determined by realtime Q-PCR. mRNA levels are relative to those of V7 (set as 100). Each data point represents the mean \pm SD ( $n=3$ ). (C) Developmental changes in hemolymph ecdysteroid concentration. Hemolymph was collected from female larvae every $6 \mathrm{~h}$ from the beginning of V5 photophase. Concentrations are expressed in $\mu \mathrm{M} 20 \mathrm{E}$-equivalents. The right ordinate is for open circles, which are an expansion of the closed-circle data points (left ordinate). Each data point represents the mean $\pm \operatorname{SD}(n=5)$.

Real-time Q-PCR analysis provides more accurate information on messenger RNA (mRNA) levels than RT-PCR. As there were no differences in the expression profiles of the three ANX IX isoforms in the ASGs (Fig. 2A), we employed real-time Q-PCR to determine the changes in ANX IX mRNA levels in the ASGs during the fifth instar stage (Fig. 2B).
Levels were high throughout the feeding period until V6, after which they declined sharply on V7, the day following gut purge, and remained low thereafter. This profile supports the data obtained by RT-PCR (Fig. 2). It should be noted that large standard deviation (SD) bars, such as that associated with the V6 data point, are frequently observed 
for data obtained during the transient phase of various developmental phenomena.

ANX IX expression profiles in the silk glands appeared to correspond to the changes in hemolymph ecdysteroid titer. Accordingly, we measured hemolymph ecdysteroid concentrations in $20 \mathrm{E}$ equivalents (Fig. 2C). The titer gradually increased from $0.018 \mu \mathrm{M}$ at $0 \mathrm{~h}$ of $\mathrm{V} 5$ to $0.3 \mu \mathrm{M}$ at 15 $\mathrm{h}$ of $\mathrm{V} 7$, and increased further until $0 \mathrm{~h}$ of $\mathrm{V} 9$. It remained at a high level for the next $18 \mathrm{~h}$, until $18 \mathrm{~h}$ of $\mathrm{V} 9$ (highest concentration: $2.89 \mu \mathrm{M}$ at $12 \mathrm{~h}$ of $\mathrm{V} 9$ ), and then sharply decreased to a low level at $0 \mathrm{~h}$ of $\mathrm{V} 10$ photophase.

\section{Response of ANX IX to 20E}

To address the issue of whether $A N X I X$ could be under the control of $20 \mathrm{E}$, we applied $1 \mu \mathrm{M} 20 \mathrm{E}$ to V7 ASGs, as these conditions had previously been shown to be sufficient to induce PCD (Terashima et al., 2000). Although the ANX IX mRNA level increased during incubation of V7 ASGs in medium alone, it was up-regulated more than two-fold after $4 \mathrm{~h}$ incubation with $1 \mu \mathrm{M} 20 \mathrm{E}$ (Figs. 3A, 4B). Expression fell to control levels after $8 \mathrm{~h}$. RT-PCR analysis showed that ANX IX-A mRNA levels decreased after $8 \mathrm{~h}$, while $B$ and $C$ isoforms were maintained at the up-regulated level (Fig. $3 \mathrm{~B})$. The addition of $\mathrm{CHX}$ suppressed the decrease in expression at $24 \mathrm{~h}$ (Fig. 3B). The concentration-response of ANX IX to 20E revealed that the gene was stimulated maximally by $1 \mu \mathrm{M} 20 \mathrm{E}$ (Fig. $3 \mathrm{C}$ ). The rapid response and the inhibition of down-regulation by $\mathrm{CHX}$ indicate that $A N X I X$ might be induced directly by $20 \mathrm{E}$.

To examine the effects of $20 \mathrm{E}$ on ANX IX expression on various days of the fifth instar stage, ASGs were incubated with or without $1 \mu \mathrm{M} 20 \mathrm{E}$ for $4 \mathrm{~h}$ (Fig. 4). Throughout this period, $A N X I X-B$ was shown to be the predominant isoform, irrespective of the presence or absence of $20 \mathrm{E}$, with the exception of V6 ASGs, in which isoforms $B$ and $C$ were equally expressed. Real-time Q-PCR revealed that $A N X I X$ did not respond to $20 \mathrm{E}$ before V6 (Fig. 4B), while it was stimulated on V7 and V8, and down-regulated on V9. ANX IX mRNA levels increased in medium alone, but were greatly enhanced by $20 \mathrm{E}$ in $\mathrm{V} 7$ and V8 ASGs. ANX IX mRNA levels in V6 ASGs did not change following incubation in medium alone or in the presence of $20 \mathrm{E}$.

\section{ANX IX response to $0.1 \mu \mathrm{M} 20 \mathrm{E}$}

The above results suggested that $\mathrm{V} 6$ is a critical day in the response of $A N X I X$ to $20 \mathrm{E}$. As the hemolymph ecdysteroid concentration on V6 was around $0.1 \mu \mathrm{M}$ (Fig. 2C), we examined the effects of $0.1 \mu \mathrm{M} 20 \mathrm{E}$ on $A N X I X$ expression in the ASGs obtained on V5, V6, and V7 (Fig. 5). In V5 ASGs, ANXIX mRNA levels increased during the initial $4 \mathrm{~h}$, with or without $20 \mathrm{E}$, suggesting that this resulted from the liberation of the ASGs from endogenous ecdysteroids. Expression decreased after $4 \mathrm{~h}$ incubation, and at $8 \mathrm{~h}$ the mRNA level in the presence of $20 \mathrm{E}$ was significantly lower than in the absence of $20 \mathrm{E}$, indicating that the suppressive effects of $20 \mathrm{E}$ took at least $4 \mathrm{~h}$ to manifest. Similar results were observed for V6 ASGs. By contrast, ANX IX mRNA levels in V7 ASGs continuously increased until $8 \mathrm{~h}$ of incubation, irrespective of the presence or absence of $20 \mathrm{E}$, indicating lower sensitivity of $A N X I X$ to $20 \mathrm{E}$ than in V5 or V6 ASGs.

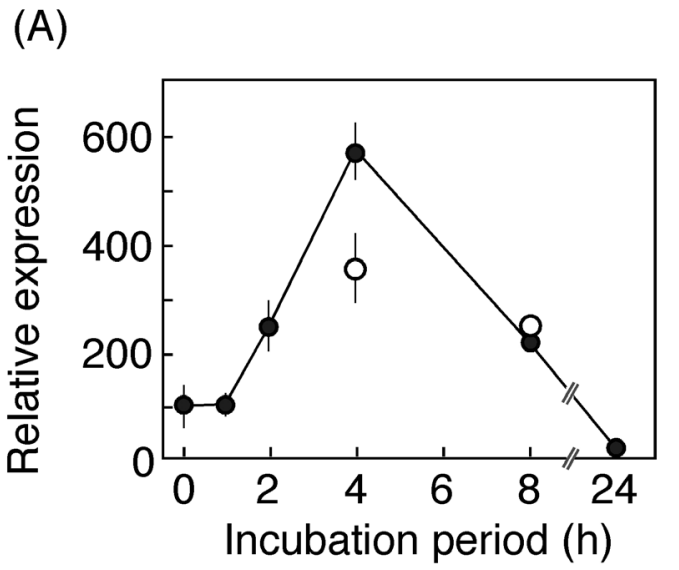

(B)

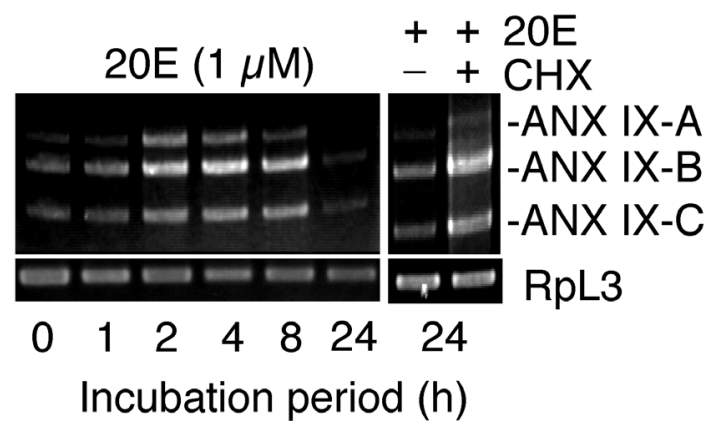

(C)

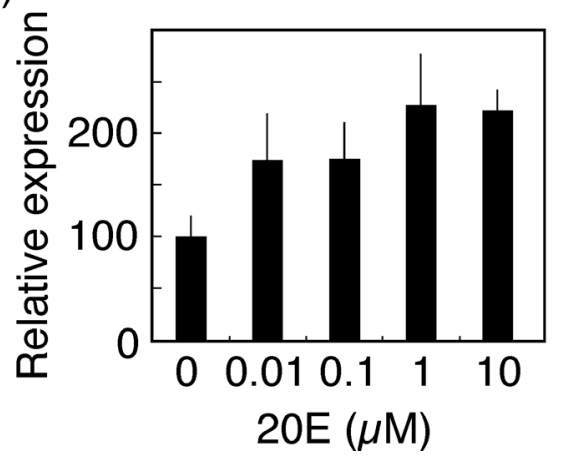

Fig. 3. Response of $A N X I X$ to $20 \mathrm{E}$ in V7 ASGs. (A) Expression profile of $A N X I X$ in V7 ASGs incubated in the presence (solid symbols) or absence (open symbols) of $1 \mu \mathrm{M} 20 \mathrm{E}$, as determined by real-time Q-PCR. Expression levels are relative to that at $0 \mathrm{~h}$ (set as 100). (B) Expression levels of $A N X I X$ isoforms as determined by RT-PCR. Left panel: V7 ASGs were incubated with $1 \mu \mathrm{M} 20 \mathrm{E}$ for the indicated time periods. Right panel: V7 ASGs were incubated with $20 \mathrm{E}$ in the presence (+) or absence (-) of $10 \mu \mathrm{g} / \mathrm{ml} \mathrm{CHX}$ for $24 \mathrm{~h}$. (C) Concentration-response of $A N X I X$ isoforms to 20E. V7 ASGs were incubated with various concentrations of $20 \mathrm{E}$ for $2 \mathrm{~h}$, based on the data shown in (A). ANX IX mRNA levels were quantitatively determined by real-time Q-PCR. Expression levels are relative to those in the ASGs incubated in medium alone (set as 100). Each data point in $A$ and $C$ represents the mean $\pm S D(n=3)$.

\section{Expression profiles of EcR and USP in vivo}

As in vivo expression of $A N X$ appears to be down-regulated by $20 \mathrm{E}$, which elicits its effects via binding with $\mathrm{EcR} /$ USP, we examined the temporal expression profiles of $E c R$ and USP. ECR-A was the predominant isoform throughout 
(A)

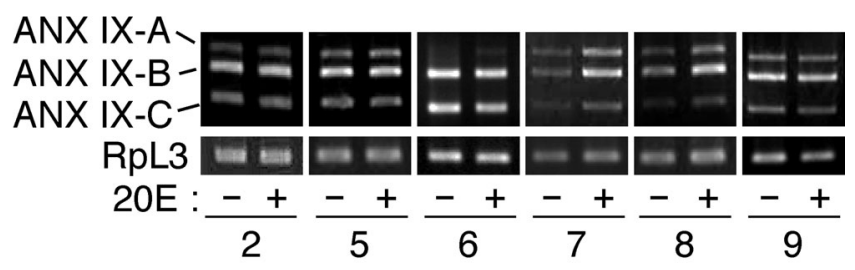

(B)

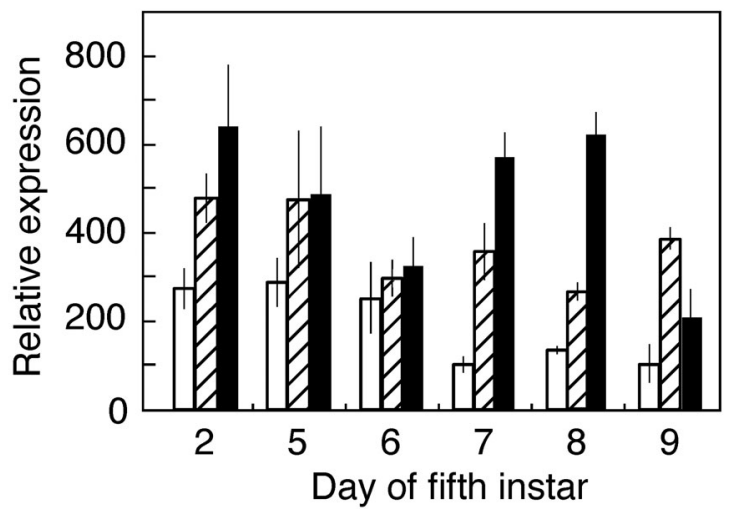

Fig. 4. Developmental changes in the response of $A N X I X$ to $20 \mathrm{E}$. ASGs obtained on various days of the fifth instar stage were incubated in the presence (+) or absence (-) of $1 \mu \mathrm{M} 20 \mathrm{E}$ for $4 \mathrm{~h}$. (A) RT-PCR analysis of individual $A N X I X$ isoforms. (B) $A N X I X$ expression levels measured by real-time Q-PCR. ANX IX mRNA levels are relative to those in freshly dissected V7 ASGs (set as 100). Open bars, before incubation; hatched bars, incubation in medium alone; solid bars, incubation with $20 \mathrm{E}$. Each data point represents the mean $\pm S D(n=3)$.

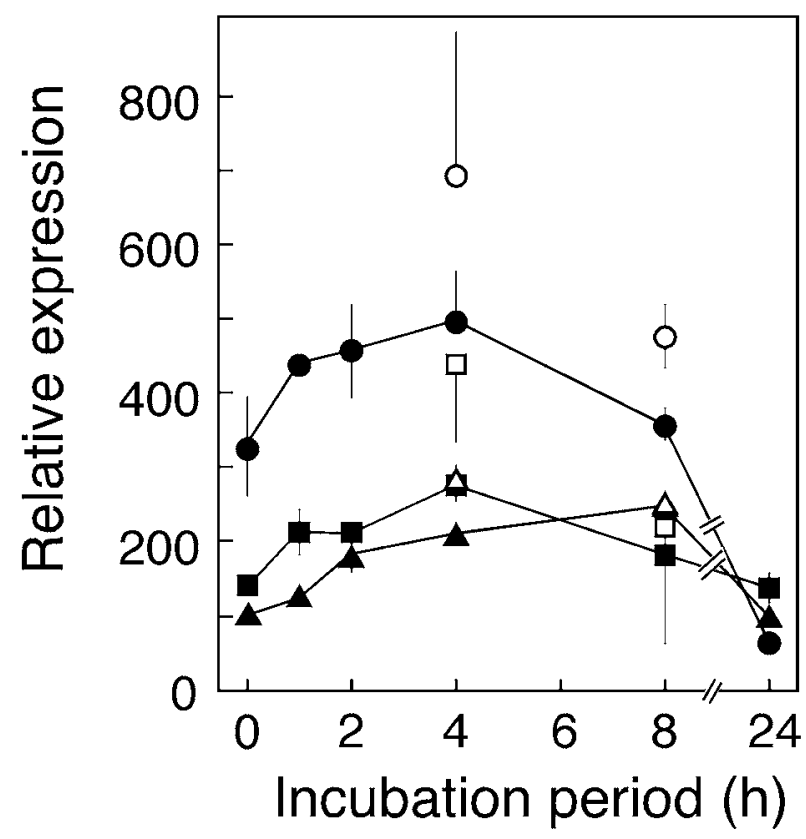

Fig. 5. Inhibition of $A N X I X$ by $0.1 \mu \mathrm{M}$ 20E. ASGs obtained on V5 (circles), V6 (squares), and V7 (triangles) were incubated with (solid symbols) or without (open symbols) $0.1 \mu \mathrm{M} \mathrm{20E}$ for the indicated time periods. Each data point represents the mean $\pm S D(n=3)$. the fifth instar stage, except on V6 (Fig. 6A). On this day, $E c R-B 1$ was up-regulated to the level of EcR-A (Fig. 6A, inset). $E c R-A$ was increased on V7 when the ecdysteroid titer began to rise, while $E c R-B 1$ remained at a similar level to that observed on V6. USP-1 expression reached a peak on V6, followed by a sharp decline on V7 (Fig. 6B). USP-2 expression remained low throughout the fifth instar stage.

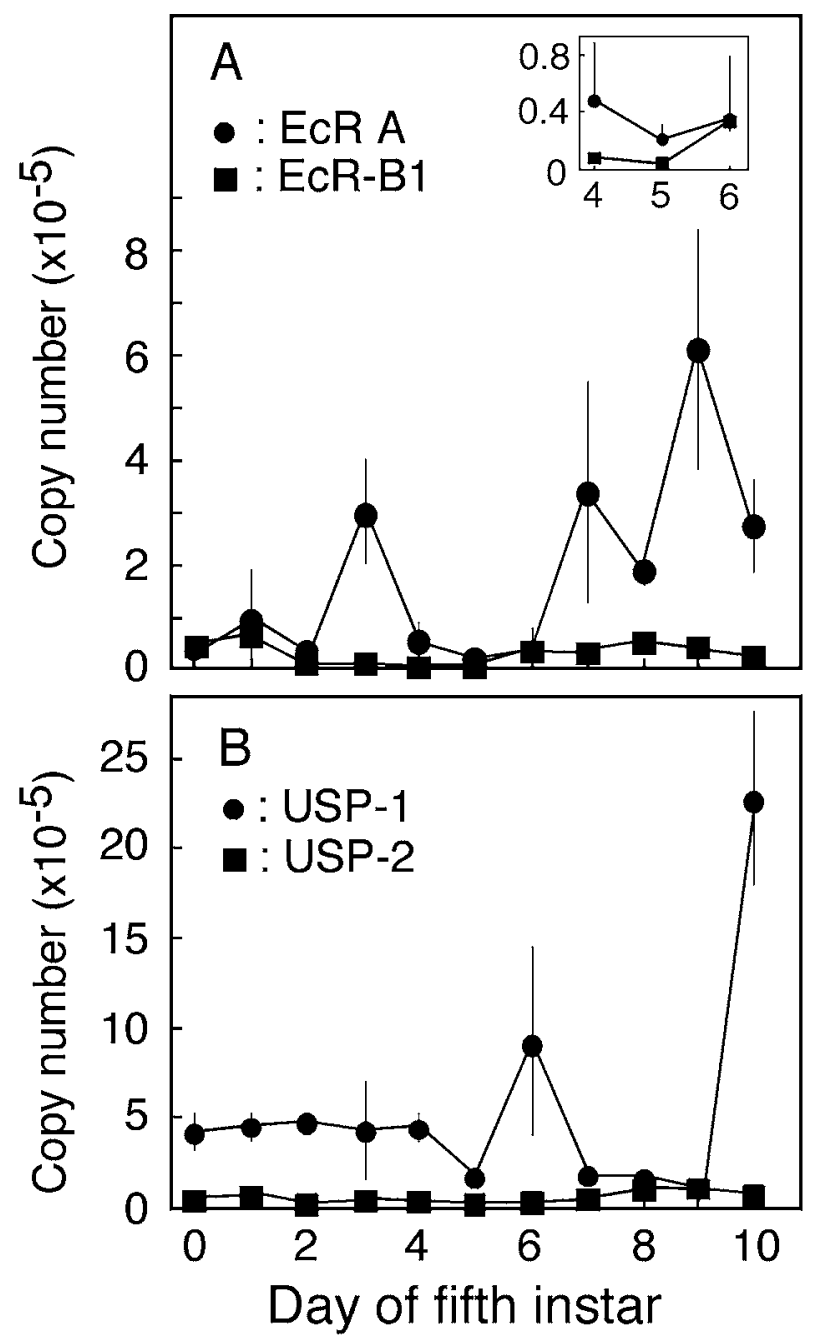

Fig. 6. Developmental profiles of (A) $E c R$ and (B) USP expression in ASGs. Total RNA was extracted daily, and EcR and USP expression levels were quantitatively determined by real-time Q-PCR. Inset of $(A)$ : an enlargement of the $\mathrm{V} 4$ to $\mathrm{V} 6$ data points. Each data point represents the mean $\pm S D(n=3)$.

\section{Changes in EcR and USP responses to 20E during the fifth instar stage}

To examine the developmental changes in the response of $E c R$ and USP to $20 \mathrm{E}$, ASGs obtained on various days of the fifth instar stage were incubated for $4 \mathrm{~h}$ in the presence or absence of $1 \mu \mathrm{M} 20 \mathrm{E}$. EcR-A and EcR-B1 were equally up-regulated by $20 \mathrm{E}$ on all days examined (Fig. 7A), but the intensity of response of each isoform to $20 \mathrm{E}$ varied with the extent of larval growth. Both isoforms were greatly stimulated on V5 and V8, but EcR-B1 was stimulated more than $E C R-A$ on $\mathrm{V} 6$ and $\mathrm{V} 7$. In addition, the ratio of the expression 
(A)
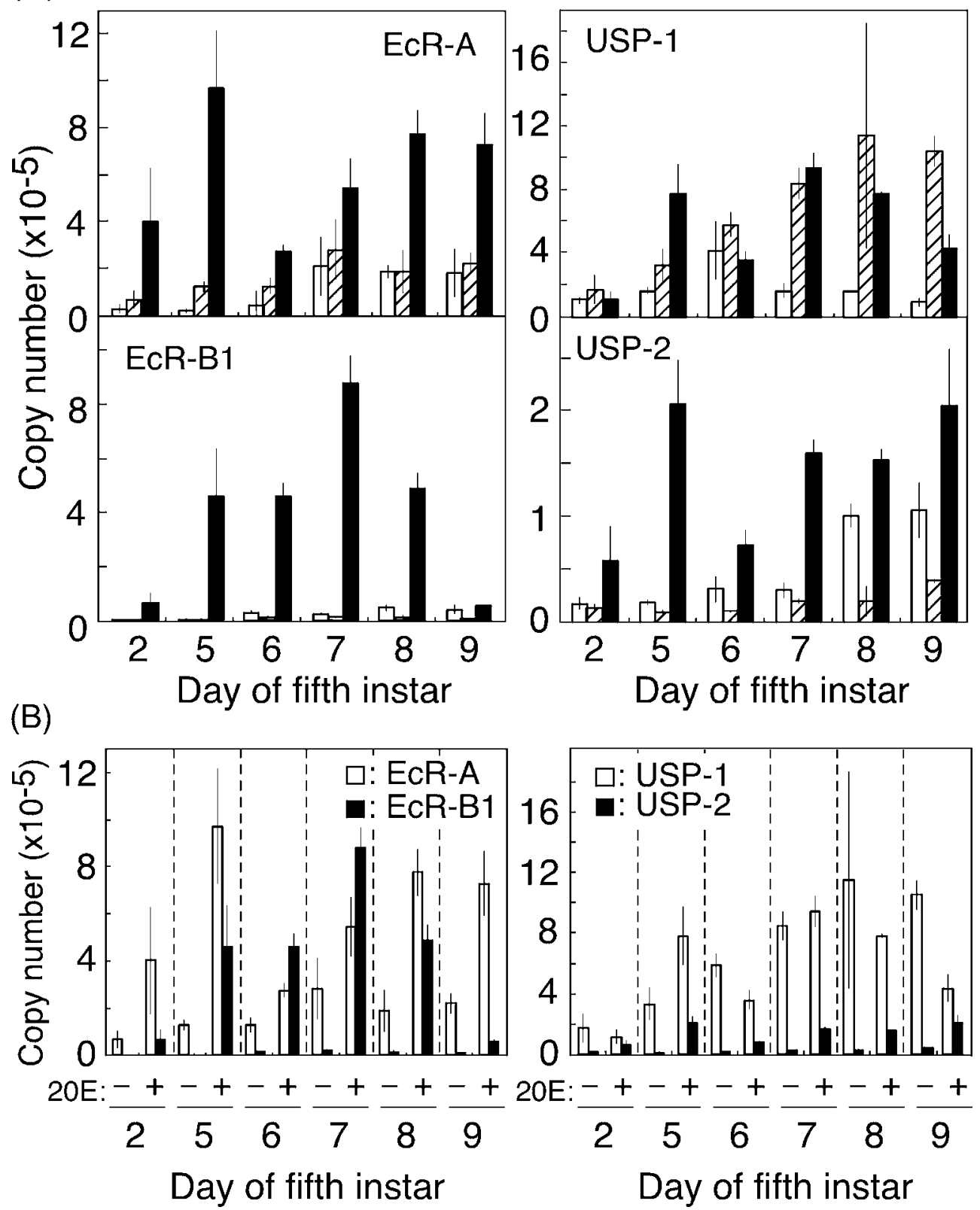

Fig. 7. Developmental changes in responses of $E C R$ and USP to 20E. ASGs obtained on various days of the fifth instar stage were incubated with or without $1 \mu \mathrm{M} 20 \mathrm{E}$ for $4 \mathrm{~h}$. (A) mRNA levels of individual isoforms were measured by real-time Q-PCR. Open bars, before incubation; hatched bars, incubation in medium alone; solid bars, incubation with 20E. (B) Comparison of expression levels between $E c R-A$ and $E c R-B 1$ (left panel), and between USP-1 and USP-2 (right panel). Data were extracted from the results shown in (A). Each data point represents the mean $\pm S D(n=3)$.

levels of $E c R-A$ to $E c R-B 1$ altered during development (Fig. 7B). EcR-A was predominantly expressed in $\mathrm{V} 2, \mathrm{~V} 5$, V8, and V9 ASGs, while ECR-B1 predominated on V6 and V7.

$20 \mathrm{E}$ did not affect the expression of USP-1 during the fifth instar stage, except for V5 ASGs, in which levels were two-fold greater than those in the control. By contrast, USP2 was consistently up-regulated by $20 \mathrm{E}$. USP-1 and USP-2 exhibited different responses to in vitro incubation. USP-1 expression was unaffected by incubation in medium alone before V7, but increased on and after V7, indicating that it might be suppressed by $20 \mathrm{E}$ and providing a possible expla- nation for the low levels of in vivo expression observed on V7 to V9. USP-2 expression was not affected by incubation in medium alone, showing that it always requires $20 \mathrm{E}$ stimulation. Although the in vitro USP isoform responses to $20 \mathrm{E}$ varied over the course of development, the expression level of USP-1 was consistently higher than USP-2 after $4 \mathrm{~h}$ incubation with $1 \mu \mathrm{M} 20 \mathrm{E}$ (Fig. 7B). It is of interest that the expression profile of $E C R-A$ following incubation with $20 \mathrm{E}$ is similar to that of USP-2, and that ECR-B1 and USP-1 also demonstrated marked similarities in expression patterns.

In order to examine the concentration-responses of $E c R$ 


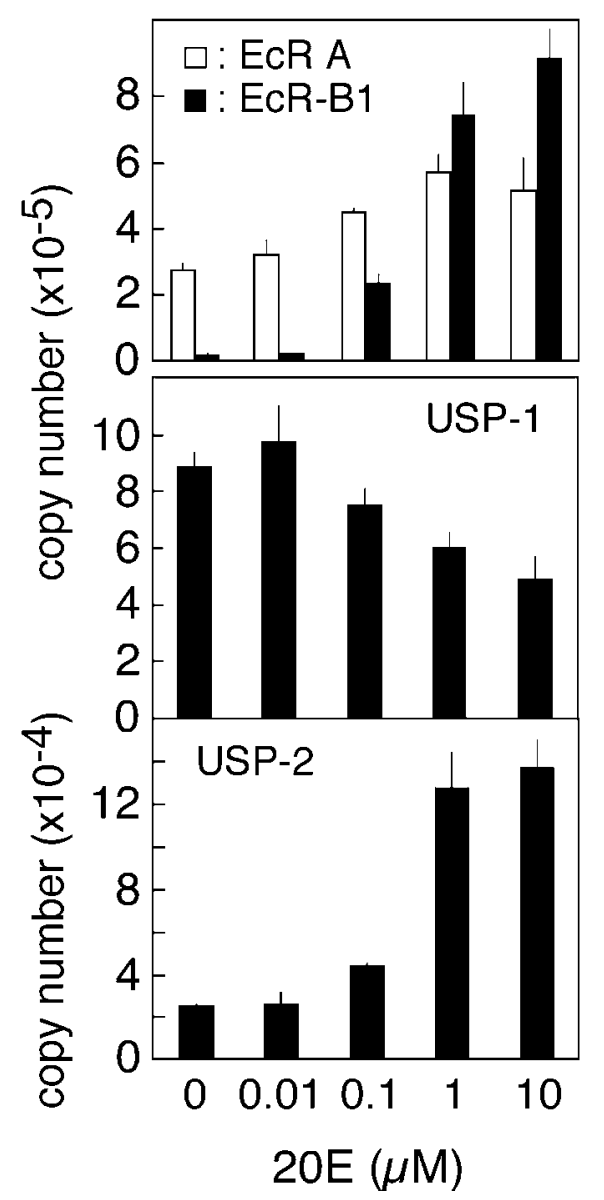

and USP to 20E, V7 ASGs were incubated with various concentrations of $20 \mathrm{E}$ for $4 \mathrm{~h}$ (Fig. 8). EcR-A was stimulated at $20 \mathrm{E}$ concentrations between 0.1 and $10 \mu \mathrm{M}$, but the stimulated level at $1 \mu \mathrm{M}$ was only twice as high as the control ( 0 $\mu \mathrm{M} 20 \mathrm{E})$. By contrast, EcR-B1 was stimulated maximally at $10 \mu \mathrm{M}$ to a level 46 -fold higher than that of the control. Interestingly, $0.1 \mu \mathrm{M} 20 \mathrm{E}$ stimulated $E c R-B 1$ to a high level but only slightly stimulated $E C R-A$. USP-1 was suppressed by $20 \mathrm{E}$ concentrations of $0.1 \mu \mathrm{M}$ and above, while, conversely, USP-2 was up-regulated at and above this concentration.

\section{EcR and USP responses to $0.1 \mu \mathrm{M} 20 \mathrm{E}$}

EcR-A mRNA levels in V5 ASGs increased after $2 \mathrm{~h}$ incubation with $0.1 \mu \mathrm{M} 20 \mathrm{E}$, and this persisted until $24 \mathrm{~h}$ (Fig. 9). By contrast, no such stimulation was observed in V6 ASGs, while expression levels in V7 ASGs decreased from 1 to $8 \mathrm{~h}$ of incubation. EcR-B1 in V5 ASGs was transiently up-regulated after $4 \mathrm{~h}$ incubation and decreased thereafter. EcR-B1 in V6 and V7 ASGs was similarly up-regulated at $4 \mathrm{~h}$, although the levels were maintained until $24 \mathrm{~h}$.

Both USP-1 and USP-2 expression levels in V5 ASGs increased until $8 \mathrm{~h}$ of incubation with 20E. In V6 ASGs, USP-1 was sharply up-regulated $1 \mathrm{~h}$ after $20 \mathrm{E}$ stimulation then down-regulated following a mild fluctuation between 1 and $4 \mathrm{~h}$. USP-2 in V6 ASGs exhibited a similar response to

Fig. 8. Concentration-responses of $E C R$ and USP isoforms to $20 \mathrm{E}$. V7 ASGs were incubated with various concentrations of $20 \mathrm{E}$ for $4 \mathrm{~h}$. mRNA levels of individual isoforms were measured by real-time $Q$ PCR. Each data point represents the mean \pm SD $(n=3)$.
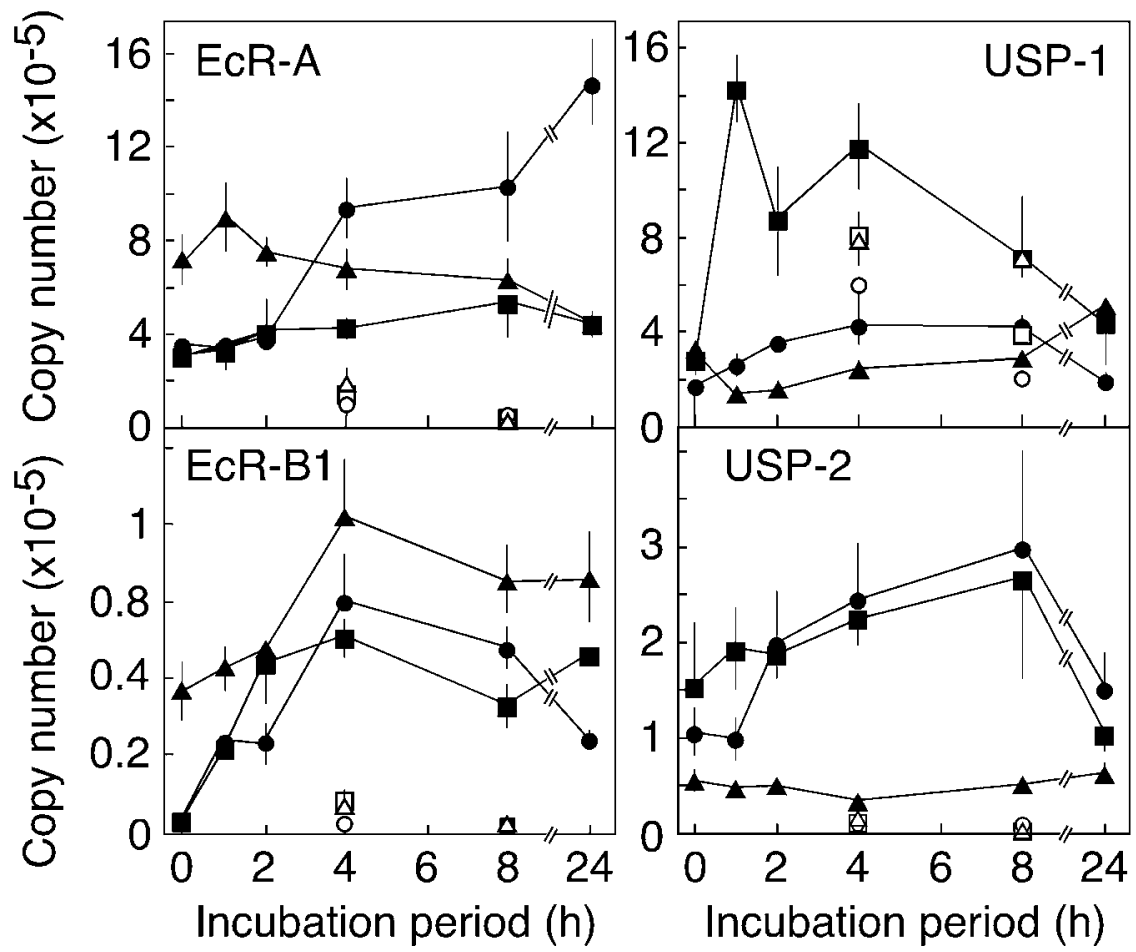

Fig. 9. Stage-specific responses of $E c R$ and USP isoforms to $0.1 \mu \mathrm{M} 20 \mathrm{E}$. ASGs obtained on V5 (circles), V6 (squares), and V7 (triangles) were incubated with (solid symbols) or without (open symbols) $0.1 \mu \mathrm{M} 20 \mathrm{E}$ for the indicated time periods. Each data point represents the mean $\pm \operatorname{SD}(n=3)$. 
$20 \mathrm{E}$ as that observed on V5. V7 USP-1 mRNA levels transiently decreased, recovered after $8 \mathrm{~h}$ of incubation, and showed a further increase thereafter. By contrast, USP-2 in V7 ASGs was unaffected by $20 \mathrm{E}$.

\section{DISCUSSION}

In Malpighian tubules and testes that survive pupal metamorphosis, high $A N X I X$ expression levels are maintained throughout the fifth instar stage. By contrast, $A N X I X$ mRNA levels in the three parts of the silk glands that undergo PCD decline as the ecdysteroid titer rises (Fig. 2A). ANX proteins are known to be involved in apoptosis in mammals and nematodes (Nakamura et al., 1998; Arur et al., 2003). As $20 \mathrm{E}$ triggers PCD of the silk glands, the reciprocal expression of $A N X I X$ and the change in hemolymph ecdysteroid titer suggest a relationship between ANX IX and 20Einduced PCD.

Before V6, ASGs are incapable of responding to $20 \mathrm{E}$ in vitro, but they gain this ability on V6 while undergoing PCD (Kakei et al., 2005). In vitro and in vivo data on ANX IX (Fig. 2A, 2B, Fig. 4)and EcR expression (Fig. 6, Fig. 7) show that $\mathrm{V} 6$ is the critical day of transition for these genes. Indeed, ANX IX mRNA levels in ASGs decrease over the period from $V 6$ to V7.

ANX V, a mammalian ANX closely involved in the control of apoptosis, reduces proteolytic activation of caspase-3 in the CEM human T-cell line, thereby suppressing epotosideinduced apoptosis (Gidon-Jeangirard et al., 1999b). In a Jurkat T-lymphocyte cell line, ANX V inhibits the formation of apoptotic bodies induced by an anti-Fas antibody (Kenis et al., 2004) and binds to the active form of protein kinase $C$ (PKC), thus inhibiting its activity (Dubois et al., 1998; CardóVila et al., 2003). In B. mori ASGs, 20E activates PKC and caspase-3 in 20E-stimulated V7 ASGs (Iga and Sakurai, unpublished data). The current study shows that $A N X I X$ is down-regulated in V6 ASGs that undergo PCD in response to $20 \mathrm{E}$ in vitro (Fig. 2A, 2B). If ANX IX has an inhibitory effect in ASG cells, similar to the role of ANX V in mammalian cells, it might suppress the 20E-dependent activation of PKC and caspase- 3 activation before $\mathrm{V} 6$, thereby inhibiting the gland's response to 20E. The down-regulation of $A N X I X$ on V6 would release the ASGs from this inhibited state, enabling them to respond to 20E while undergoing PCD.

The $A N X I X$ expression level remains low after gut purge when the hemolymph ecdysteroid titer is high, suggesting that $A N X I X$ is suppressed by $20 \mathrm{E}$. During the period from $\mathrm{V} 5$ to $\mathrm{V} 7$, the titer ranges from 0.01 to $0.1 \mu \mathrm{M}$ (Fig. $2 \mathrm{C}$ ) and it is possible that the down-regulation of $A N X I X$ is caused by this small increase in ecdysteroid concentration. This is supported by our in vitro data (Fig. 5) showing that ANX IX mRNA levels increase when V5 to V7 ASGs are incubated in a $20 \mathrm{E}$-free medium, in which $A N X I X$ is liberated from the inhibitory effects of $20 \mathrm{E}$.

The $20 \mathrm{E}$ signal is mediated through a heterodimeric nuclear-receptor complex comprising EcR and USP. The change in abundance of EcR and USP isoforms occurs before pupal metamorphosis (Talbot et al., 1993; Bender et al., 1997; Song and Gilbert, 1998), and the specific combination of isoforms is important in inducing particular developmental events. The dominant expression of EcR-B1 at the onset of pupal metamorphosis mediates the $20 \mathrm{E}$ signal for puparium forma- tion in most tissues of $D$. melanogaster, except for the salivary gland (Bender et al., 1997; Davis et al., 2005). Both loss-offunction EcR-A and EcR-B1 mutants demonstrate PCD suppression in the salivary glands, indicating that both isoforms are indispensable for mediating the $20 \mathrm{E}$ signal triggering PCD, although it is unclear whether the relative EcR isoform titers are of importance (Cherbas et al., 2003).

In the ASGs, ECR-A is predominantly expressed throughout the fifth instar stage, except on V6 when EcR-B1 is up-regulated by $20 \mathrm{E}$ to reach similar expression levels to $E c R-A$ (Fig. 6A). Up-regulation of $E c R-B 1$ is lower than $E c R$ $A$ on other days of the fifth instar stage (Fig. 7B). The transient change in the ratio of $E c R$ isoform titers on $V 6$ is likely to be associated with the change in $E c R$ gene response to $20 \mathrm{E}$ on the same day.

USP-1 in the ASGs is also transiently up-regulated on V6 (Fig. 6B). The small increase in hemolymph ecdysteroid titer $(0.1$ to $0.2 \mu \mathrm{M})$ around $\mathrm{V} 6$ might account for this peak, as USP-1 is sharply up-regulated by $0.1 \mu \mathrm{M}$ but not $1 \mu \mathrm{M}$ $20 \mathrm{E}$, which occurs only in V6 ASGs (Fig. 9, Fig 7A). The responses of USP-1 and USP-2 to $20 \mathrm{E}$ differ from each other and are stage specific (Fig. 7B). In Manduca sexta prothoracic glands, the predominant isoform of USP alters from USP-1 to USP-2 in association with the larvae entering the prepupal period (Song and Gilbert, 1998). The stagespecific and hormone concentration-specific responses of individual USP isoforms to $20 \mathrm{E}$, also seen in B. mori ASGs, indicate the importance of the $\mathrm{V} 6$ peak, although the role of USP in PCD of the ASGs remains to be elucidated.

Gene expression is controlled by $20 \mathrm{E}$ via binding the 20E-EcR/USP complex to its response element, EcRE. In the $M$. sexta GV1 cell line, 20E stimulation of $M H R-3$ expression involves the nuclear-receptor complex EcR-B1/ USP-1 binding to EcRE1 of the MHR-3 gene. In contrast, EcR-A/USP-2 suppresses MHR-3 expression (Lan et al., 1999; Hiruma and Riddiford, 2004). A search for the ANX IX EcRE in the $B$. mori genomic database revealed four putative elements within the 5' flanking region, suggesting that ANX IX gene expression might be under the control of EcR isoforms, in a similar manner to MHR-3. This is also supported by the fact that ASG ANX IX mRNA levels decrease from $V 6$ to $V 7$, in line with the transition of $E c R$ isoform ratio titers (Fig. 7B).

Based on these findings, we conclude that ANX IX acts as an inhibitor of 20E-induced PCD, as it persists at high levels in tissues that survive metamorphosis, but the levels are decreased in tissues that undergo PCD. We suggest that ANX IX participates in the acquisition of ASG responsiveness to $20 \mathrm{E}$ on $\mathrm{V} 6$ of the fifth instar stage by delaying or suppressing the $20 \mathrm{E}$ signaling pathway.

\section{ACKNOWLEDGMENTS}

We thank Dr. S. Harada of the Medical School, Kanazawa University, for critical advice on real-time Q-PCR. We are grateful to Mr. T. Sekimoto for collecting larval hemolymph for ecdysone RIA. This study was supported by a Grant-in-Aid for Scientific Research from the Japan Society for the Promotion of Science to S.S.

\section{REFERENCES}

Abrams JM (1999) An emerging blueprint for apoptosis in Drosophila. Trends Cell Biol 9: 435-440 
Arur S, Uche UE, Rezaul K, Fong M, Scranton V, Cowan AE, Mohler W, Han DK (2003) Annexin I is an endogenous ligand that mediates apoptotic cell engulfment. Dev Cell 4: 587-598

Bender M, Imam FB, Talbot WS, Ganetzky B, Hogness DS (1997) Drosophila ecdysone receptor mutations reveal functional differences among receptor isoforms. Cell 91: 777-788

Cardó-Vila M, Arap W, Pasqualini R (2003) Alpha v beta 5 integrindependent programmed cell death triggered by a peptide mimic of annexin V. Mol Cell 11: 1151-1162

Cherbas L, Hu X, Zhimulev I, Belyaeva E, Cherbas P (2003) EcR isoforms in Drosophila: testing tissue-specific requirements by targeted blockade and rescue. Development 130: 271-284

Chinzei Y (1975) Induction of histolysis by ecdysterone in vitro: breakdown of anterior silk gland in silkworm, Bombyx mori (Lepidoptera: Bombycidae). Appl Entomol Zool (Jpn) 10: 136-138

Chomczynski P, Sacchi N (1987) Single-step method of RNA isolation by acid guanidinium thiocyanate-phenol-chloroform extraction. Anal Biochem 162: 156-159

Davidson FF, Dennis EA, Powell M, Glenney JR Jr (1987) Inhibition of phospholipase A2 by "lipocortins" and calpactins. An effect of binding to substrate phospholipids. J Biol Chem 262: 1698-1705

Davis MB, Carney GE, Robertson AE, Bender M (2005) Phenotypic analysis of $E c R-A$ mutants suggests that $E c R$ isoforms have unique functions during Drosophila development. Dev Biol 282: 385-396

Dubois T, Mira JP, Feliers D, Solito E, Russo-Marie F, Oudinet JP (1998) Annexin $V$ inhibits protein kinase $C$ activity via a mechanism of phospholipid sequestration. Biochem J 330: 12771282

Emans N, Gorvel JP, Walter C, Gerke V, Kellner R, Griffiths G, Gruenberg J (1993) Annexin II is a major component of fusogenic endosomal vesicles. J Cell Biol 120: 1357-1369

Gidon-Jeangirard C, Solito E, Hofmann A, Russo-Marie F, Freyssinet JM, Mart'nez MC (1999a) Annexin V counteracts apoptosis while inducing $\mathrm{Ca}^{2+}$ influx in human lymphocytic $\mathrm{T}$ cells. Biochem Biophys Res Commun 265: 709-715

Gidon-Jeangirard C, Hugel B, Holl V, Toti F, Laplanche JL, Meyer $D$, Freyssinet JM (1999b) Annexin $V$ delays apoptosis while exerting an external constraint preventing the release of $\mathrm{CD}^{+}$ and $\mathrm{PrPc}^{+}$membrane particles in a human $\mathrm{T}$ lymphocyte model. J Immunol 162: 5712-5718

Gilbert LI, Rybczynski R, Tobe SS (1996) Endocrine cascade in insect metamorphosis. In "Metamorphosis" Ed by LI Gilbert, JR Tata, BG Atkinson, Academic Press, San Diego, pp 59-107

Hiruma K, Riddiford LM (2004) Differential control of MHR3 promoter activity by isoforms of the ecdysone receptor and inhibitory effects of E75A and MHR3. Dev Biol 272: 510-521

Jacobson MD, Weil M, Raff MC (1997) Programmed cell death in animal development. Cell 88: 347-354

Jiang C, Lamblin AF, Steller H, Thummel CS (2000) A steroid-triggered transcriptional hierarchy controls salivary gland cell death during Drosophila metamorphosis. Mol Cell 5: 445-455

Kakei M, Iwami M, Sakurai S (2005) Death commitment in the anterior silk gland of the silkworm, Bombyx mori. J Insect Physiol 51: 17-25

Kamimura M, Tomita S, Fujiwara H (1996) Molecular cloning of an ecdysone receptor (B1 isoform) homologue from the silkworm, Bombyx mori, and its mRNA expression during wing disc development. Comp Biochem Physiol B Biochem Mol Biol 113: 341-347

Kamimura M, Tomita S, Kiuchi M, Fujiwara H (1997) Tissue-specific and stage-specific expression of two silkworm ecdysone receptor isoforms - ecdysteroid-dependent transcription in cultured anterior silk glands. Eur J Biochem 248: 786-793

Kenis $H$, van Genderen $H$, Bennaghmouch A, Rinia HA, Frederik $P$, Narula J, Hofstra L, Reutelingsperger CP (2004) Cell surfaceexpressed phosphatidylserine and annexin $\mathrm{A} 5$ open a novel portal of cell entry. J Biol Chem 279: 52623-52629
König J, Prenen J, Nilius B, Gerke V (1998) The annexin II-p11 complex is involved in regulated exocytosis in bovine pulmonary artery endothelial cells. J Biol Chem 273: 19679-19684

Lan Q, Hiruma K, Hu X, Jindra M, Riddiford LM (1999) Activation of a delayed-early gene encoding MHR3 by the ecdysone receptor heterodimer EcR-B1-USP-1 but not by EcR-B1-USP-2. Mol Cell Biol 19: 4897-4906

Lee CY, Baehrecke EH (2001) Steroid regulation of autophagic programmed cell death during development. Development 128: 1443-1455

Matsunaga TM, Fujiwara $\mathrm{H}$ (2002) Identification and characterization of genes abnormally expressed in wing-deficient mutant (flügellos) of the silkworm, Bombyx mori. Insect Biochem Mol Biol 32: 691-699

Matsuoka T, Fujiwara H (2000) Expression of ecdysteroid-regulated genes is reduced specifically in the wing discs of the wing-deficient mutant (fl) of Bombyx mori. Dev Genes Evol 210: 120-128

Mira JP, Dubois T, Oudinet JP, Lukowski S, Russo-Marie F, Geny B (1997) Inhibition of cytosolic phospholipase A2 by annexin $V$ in differentiated permeabilized HL-60 cell. J Biol Chem 272: 10474-10482

Nakamura N, Ban T, Yamaji K, Yoneda Y, Wada Y (1998) Localization of the apoptosis-inducing activity of lupus anticoagulant in an annexin V-binding antibody subset. J Clin Invest 101: 19511959

Rescher U, Gerke V (2004) Annexins - unique membrane binding proteins with diverse functions. J Cell Sci 117: 2631-2639

Sakurai S (1983) Temporal organization of endocrine events underlying larval-larval ecdysis in the silkworm, Bombyx mori. J Insect Physiol 29: 919-932

Sakurai S (1984) Temporal organization of endocrine events underlying larval-pupal metamorphosis in the silkworm, Bombyx mori. J Insect Physiol 30: 657-664

Sakurai S Satake S, Kaya M (1998) Hemolymph ecdysteroid titer and ecdysteroid-dependent developmental events in the lastlarval stadium of the silkworm, Bombyx mori: role of low ecdysteroid titer in larval-pupal metamorphosis and a reappraisal of the head critical period. J Insect Physiol 44: 867-881

Sambrook J, Russell DW (2001) Molecular Cloning: A Laboratory Manual. 3rd Ed, Cold Spring Harbor Laboratory Press, New York, pp 6.28-6.30

Song Q, Gilbert LI (1998) Alterations in ultraspiracle (USP) content and phosphorylation state accompany feedback regulation of ecdysone synthesis in the insect prothoracic gland. Insect Biochem Mol Biol 28: 849-860

Talbot WS, Swyryd EA, Hogness DS (1993) Drosophila tissues with different metamorphic responses to ecdysone express different ecdysone receptor isoforms. Cell 73: 1323-1337

Terashima J, Yasuhara N, Iwami M, Sakurai S (2000) Programmed cell death triggered by insect steroid hormone, 20-hydroxyecdysone, in the anterior silk gland of the silkworm, Bombyx mori. Dev Genes Evol 210: 545-558

Tsuzuki S, Iwami M, Sakurai S, (2001) Ecdysteroid-inducible genes in the programmed cell death during insect metamorphosis. Insect Biochem Mol Biol 31: 321-331

Tzertzinis G, Malecki A, Kafatos FC (1994) BmCF1, a Bombyx mori RXR-type receptor related to the Drosophila ultraspiracle. J Mol Biol 238: 479-486

Xia QY, Fujii H, Kusakabe T, Banno Y (2001) Identification of three annexin IX isoforms generated by alternative splicing of the carboxyl-terminal exon in silkworm, Bombyx mori. Insect Biochem Mol Biol 32: 9-14

Yin VP, Thummel CS (2005) Mechanisms of steroid-triggered programmed cell death in Drosophila. Semin Cell Dev Biol 16: $237-243$

(Received November 24, 2005 / Accepted December 13, 2005) 